\title{
Dank an die Gutachter des Jahres 2020
}

Bei der Begutachtung und Durchsicht von Manuskripten für die RöFo haben uns im vergangenen Jahr zahlreiche Experten in Kliniken und Instituten unterstützt, die nachfolgend genannt sind und denen die Herausgeber der RöFo und der Verlag für ihre Hilfe herzlich danken.

Zunächst genannt seien die Gutachter, die für ihr besonderes Engagement ausgezeichnet wurden:

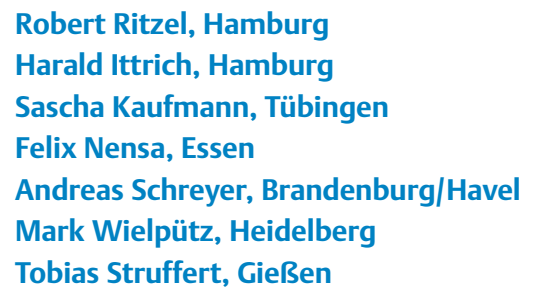

Gerhard Adam, Hamburg

Saif Afat, Tübingen

Omar M. Albtoush, Amman

Heiko Alfke, Lüdenscheid

Hatem Alkadhi, Zurich

Jens Altenbernd, Essen

Michael Amling, Hamburg

Gustav Andreisek, Zürich

Gerald Antoch, Düsseldorf

Georg Antoniadis, Gunzburg

Ivayla Apostolova, Hamburg

Evelyn Asbach, München

Patrick Asbach, Berlin

Rene Aschenbach, Jena

Ulrika Asenbaum, Wien

Maxim Avanesov, Hamburg

Bettina Baeßler, Köln

Pascal Andreas Thomas Baltzer, Wien

Fabian Bamberg, Freiburg

Christopher Bangard, Hanau

Peter Bannas, Hamburg

Jörg Barkhausen, Lübeck

Ralf W. Bauer, Wiesbaden

Thomas Baum, München

Benjamin Bender, Tübingen

Martin Bendszus, Heidelberg

Ansgar Berlis, Augsburg

Jürgen Biederer, Heidelberg

Christoph Binkert, Winterthur

Thorsten Bley, Würzburg

Karin Bock, Marburg

Malte Bongers, Tübingen

Denise Bos, Essen

Marcus Both, Kiel

Marc Brockmann, Mainz

Philipp Bruners, Aachen

Horst Brunner, Ulm

Andreas Bucher, Frankfurt
Boris Buerke, Münster

Alexander Christian Bunck, Köln

Arno Bücker, Homburg

Claus D. Claussen, Tübingen

Dirk Andre Clevert, München

Mathias Cohnen, Neuss

Adrian Curta, München

Marco Das, Aachen

Thomas Decker, Neubrandenburg

Stefan Delorme, Heidelberg

Sabine Dettmer, Hannover

Marc Dewey, Berlin

Stefan Diederich, Düsseldorf

Olaf Dietrich, München

Matthias Dietzel, Jena

Felix Doellinger, Berlin

Volker Duda, Marburg

Constantin Ehrengut, Leipzig

Tobias Engelhorn, Erlangen

Kathrin Entz, Frankfurt am Main

Birgit Ertl-Wagner, Toronto ON

Arne Estler, Tübingen

Andre Euler, Zürich

Christian von Falck, Hannover

Wolfgang Fendler, Essen

Martin Fiebich, Gießen

Jens Fiehler, Hamburg

Roman Fischbach, Hamburg

Thomas Fischer, Berlin

Cornelia Focke, Neubrandenburg

Michael Forsting, Essen

Bernd Frericks, Berlin

Björn Friebe, Magdeburg

Jan Fritz, New York

Andreas Froelich, Hamburg

Alex Frydrychowicz, Lübeck

Marco Galster, Nürnberg

Tobias Gassenmaier, Würzburg
Sebastian Gassenmaier, Tübingen

Sergios Gatidis, Tübingen

Tobias Gauer, Hamburg

Bernhard Gebauer, Berlin

Dominik Geisel, Berlin

Tobias Geith, München

Mirjam Gerwing, Münster

Lucas L. Geyer, München

Elke R. Gizewski, Innsbruck

Thoas Grieser, Augsburg

Alexander Grimm, Tübingen

Nils Große Hokamp, Köln

Hannes Gruber, Innsbruck

Andreas Gröschel, Münster

Gerd Grözinger, Tübingen

Matthias Gutberlet, Leipzig

Friedrich Götz, Hannover

Rolf Wilhelm Günther, Berlin

Christian R. Habermann, Hamburg

Thomas Hackländer, Wuppertal

Dietbert Hahn, Kürnach

Peter Hallscheidt, Heidelberg

Okka Wilkea Hamer, Regensburg

René Hartensuer, Münster

Stefan Hassfekd, Dortmund

Johannes Haubold, Essen

Lukas Hechelhammer, Sankt Gallen

Walter Heindel, Münster

Frank Oliver Gerhard Henes, Hamburg

Jeanette Henkelmann, Leipzig

Jan Philipp Hering, Ibbernbüren

Jochen Herrmann, Hamburg

Jan B. Hinrichs, Hannover

Wolfgang Hirsch, Leipzig

Karl-Titus Hoffmann, Leipzig

Christian Hoffmann, Mainz

Rüdiger Hoffmann, Tübingen

Joachim Hohmann, Basel 
Azadeh Hojreh, Wien

Marius Horger, Tübingen

Norbert Hosten, Greifswald

Stefan Hähnel, Heidelberg

Martin Härter, Hamburg

Michael Ingrisch, München

Harald Ittrich, Hamburg

Rolf Janka, Erlangen

Florian Jungmann, Mainz

Kerstin Jungnickel, Magdeburg

Manoj Kakkassery, Berlin

Birgit Kammer, München

Lars Kamper, Wuppertal

Oliver Kastrup, Essen

Hans-Ulrich Kauczor, Heidelberg

Sascha Kaufmann, Tübingen

Michael Gerhard, Kaul, Hamburg

Annika Keulers, Aachen

Daniela Kildal, Visp

Klaus Kirchhof, Jena

Julian Kirchner, Düsseldorf

Dietmar Kivelitz, Hamburg

Stephan Klebe, Essen

Bernhard Daniel Klumpp, Tübingen

Michael Knauth, Göttingen

Uwe Koch-Gromus, Hamburg

Elmar Kotter, Freiburg

Ulrich Kramer, Winnenden

Matthias Krause, Hamburg

K.-F. Kreitner, Mainz

Ilonka Kreitschmann-Andermahr, Essen

Bodo Kress, Frankfurt

Christian R. Krestan, Wien

Gabriele Anja Krombach, Gießen

Patrick Krumm, Tübingen

Heidrun Krämer, Gießen

Jan Robert Kröger, Köln

Christiane K. Kuhl, Aachen

Sabrina Kösling, Halle

Hilmar Kühl, Essen

Mark E. Ladd, Heidelberg

Heinrich Lanfermann, Hannover

Patrick Langguth, Kiel

Sönke Langner, Rostock

Simon Lennartz, Köln

Mario Liebmann, Bremen

Thomas Lindner, Hamburg

André Lollert, Mainz, Germany

Reinhard Loose, Nürnberg

Joachim Lotz, Göttingen

Martin G. Mack, München

David Maintz, Köln

Simon S. Martin, Frankfurt

Alexander Massmann, Homburg

Martin H. Maurer, Bern

Marius Mayerhoefer, Wien
Hans-Joachim Mentzel, Jena

Henrik Jakob Michaely, Karlsruhe

Peter Minko, Homburg/Saar

Walter Moeller-Hartmann, Meppen

Nuschin Morakkabati-Spitz, Bonn

Jörg Detlev Moritz, Kiel

Iris Muehlen, Erlangen

Ullrich Gerd Mueller-Lisse, München

Alexander Mundinger, Osnabrück

Sven Mutze, Berlin

Jonathan Nadjiri, München

Felix Nensa, Essen

Claus Nolte-Ernsting, Mülheim an der

Ruhr

Hannes Nordmeyer, Solingen

Jörg Oestmann, Berlin

Ahmed E. Othman, Tübingen

Stephanie Panzer, Murnau am Staffelsee

Philipp M. Paprottka, München

Kersten Peldschus, Hamburg

Tobias Penzkofer, Berlin

Philippe L. Pereira, Heilbronn

Thorsten Persigehl, Köln

Bernhard Petritsch, Würzburg

Mirko Pham, Würzburg

Claus Christian Pieper, Bonn

Daniel Pinto dos Santos, Köln

Ivan Platzek, Dresden

Heike Preibsch, Tübingen

Helmut Prosch, Wien

Michael Puesken, Köln

Azien Quitzke, Hamburg

Alexander Radbruch, Heidelberg

Marc Regier, München

Christoph Rehnitz, Heidelberg

Maximilian Reiser, München

Bernhard Renger, München

Diane Miriam Renz, Hannover

Christian O. Ritter, Göttingen

Kerstin Ritter, Berlin

Robert M. Ritzel, Hamburg

Patrik Rogalla, Berlin

Ernst J. Rummeny, München

Benedikt Schaarschmidt, Essen

Alexander Schegerer, Neuherberg

Christian Scheurig-Münkler, Augsburg

Lars Schimmöller, Düsseldorf

Kathrin Schindler, Erfurt

Marc Schlamann, Köln

Thomas Schlosser, Essen

Jörg Schmehl, Tübingen

Axel Schmid, Erlangen

Bernd Ludwig Schmitz, Ulm

Maria Schoder, Wien

Andreas G. Schreyer, Brandenburg a.d. Havel
Maximilian Franz Schulze-Hagen, Aachen

Klaus Schunk, Koblenz

Oliver Sedlaczek, Heidelberg

Ferdinand Seith, Tübingen

Malte Sieren, Lübeck

Martin Sinn, Hamburg

Gregor Sommer, Basel

Ina Sorge, Leipzig

Martin Soschynski, Freiburg

Fabian Springer, Tübingen

Georg Stamm, Hannover

Jörg Stattaus, Gelsenkirchen

Marc Steinborn, München

Wolfram Stiller, Heidelberg

Corinna Storz, Tübingen

Paul Stracke, Münster

Florian Streitparth, München

Jonas Stroeder, Homburg/Saar

Tobias Struffert, Gießen

Roland Syha, Tübingen

Wolfgang Maximilian Thaiss, Tübingen

Jens M. Theysohn, Essen

Kolja M. Thierfelder, Rostock

Christoph Gregor Trumm, München

Ilias Tsiflikas, Tübingen

Michael Uder, Erlangen

Wibke Uller, Regensburg

Tim Ullrich, Düsseldorf

Horst Urbach, Freiburg

Volker Vieth, Ibbernbüren

Jens Vogel-Claussen, Hannover

Thomas J. Vogl, Frankfurt

Dierk Vorwerk, Ingolstadt

Marlies Wagner, Frankfurt am Main

Michael Walz, Eschborn

Malte Lennart Warncke, Hamburg

Christoph Weber, Hamburg

Marc-André Weber, Rostock

Stefanie Weigel, Münster

Christina M. Wendl, Regensburg

Andreas Wenke, Münster

Axel Wetter, Essen

Stephan Wetzel, Hirslanden

Thomas Widek, Graz

Gerlig Widmann, Innsbruck

C. Wiedenroth, Bad Nauheim

Mark Oliver Wielpütz, Heidelberg

Benedikt Wiestler, München

Bernd Wintersperger, Toronto

Florian Wolf, Wien

Dag Wormanns, Berlin

Karsten Wrede, Essen

Wolfgang Wuest, Erlangen

jin Yamamura, Hamburg

Stephan Zangos, Frankfurt 